Journal for ImmunoTherapy of Cancer

Neppelenbroek SI.M, Kersten MJ, et al. Immune checkpoint inhibitor treatment and atherosclerotic cardiovascular disease: an emerging clinical problem. Journal for ImmunoTherapy of Cancer 2021;9:e002916. doi:10.1136/ jitc-2021-002916

Accepted 08 June 2021

Check for updates

(c) Author(s) (or their employer(s)) 2021. Re-use permitted under CC BY-NC. No commercial re-use. See rights and permissions. Published by BMJ.

For numbered affiliations see end of article.

Correspondence to Dr Tom T.P. Seijkens;

t.t.seijkens@amsterdamumc.nl

\title{
Immune checkpoint inhibitor treatment and atherosclerotic cardiovascular disease: an emerging clinical problem
}

\author{
Kikkie Poels, ${ }^{1}$ Suzanne I.M. Neppelenbroek, ${ }^{2,3}$ Marie José Kersten, ${ }^{3}$ \\ M. Louisa Antoni, ${ }^{4}$ Esther Lutgens, ${ }^{1,5,6}$ Tom T.P. Seijkens ${ }^{1,3,7}$
}

\begin{abstract}
Antibody-mediated blockade of co-inhibitory molecules such as cytotoxic T lymphocyte-associated protein 4, PD1 and PDL1 elicits potent antitumor responses and improves the prognosis of many patients with cancer. As these immune checkpoint inhibitors (ICls) are increasingly prescribed to a diverse patient population, a broad range of adverse effects is emerging. Atherosclerosis, a lipid-driven chronic inflammatory disease of the large arteries, may be aggravated by ICI treatment. In this review, we discuss recent clinical studies that analyze the correlation between $\mathrm{ICl}$ use and atherosclerotic cardiovascular disease (CVD). Indeed, several studies report an increased incidence of atherosclerotic CVD after $\mathrm{ICl}$ administration, with the occurrence of pathologies such as myocardial infarction, ischemic stroke and coronary artery disease significantly higher after ICl use. Increased awareness and better monitoring of ICl-treated patients can elucidate risk factors that contribute to ICl-induced aggravation of atherosclerosis and identify promising treatment strategies. For now, optimal cardiovascular risk assessment is required to protect ICl-receiving patients and long-term survivors of cancer from the detrimental effects of ICI therapy on atherosclerotic CVD.
\end{abstract}

\section{INTRODUCTION}

The growing clinical application of immune checkpoint inhibitors (ICIs) both emphasizes the unprecedented beneficial impact of ICIs on the prognosis of patients with cancer and increases our knowledge on the toxicity of these drugs. ${ }^{1-3}$ ICIs that are used in clinical practice include monoclonal antibodies that target the inhibitory immune checkpoint proteins cytotoxic $\mathrm{T}$ lymphocyte-associated protein 4 (CTLA4), programmed cell death protein 1 (PD1) and programmed cell death protein ligand 1 (PDL1). Nowadays, ICIs have been approved for more than 50 indications, and the percentage of patients with cancer eligible for ICI treatment increased from $1.54 \%$ in 2011 to $43.63 \%$ in 2018 and continues to increase rapidly. ${ }^{4}$ Although ICIs were initially approved for patients with metastasized malignancies only, they have become a cornerstone of cancer treatment in the past decade. Indications have expanded to (neo)adjuvant and maintenance settings, and ICI therapies are increasingly combined with other treatment modalities, such as chemotherapy, radiotherapy and targeted therapies by small molecule inhibitors. ${ }^{5-9}$

Immune-related adverse events (IRAEs) are a well-known toxicity of ICIs and result from a loss-of-self-tolerance, which triggers acute autoimmune-like inflammatory responses that may affect every organ. ${ }^{3}$ Severe IRAEs occur in $20 \%-50 \%$ of the ICI-treated patients and clinical manifestations include colitis, dermatitis, thyroiditis, pneumonitis, hepatitis and hypophysitis, and multiorgan involvement is frequently observed. ${ }^{3} 1011$ Cardiovascular (CV) IRAEs include myocarditis, pericarditis and vasculitis, and account for $0.39 \%, 0.30 \%$ and $0.26 \%$ of the IRAEs, respectively. ${ }^{12}$ Although relatively rare, ICIassociated myocarditis is a severe complication with a mortality rate of $27.0 \%-39.7 \% .{ }^{13} 14$ These acute CV complications, which most commonly occur within 6 weeks after initiation of ICI therapy, are pathologically characterized by immune cell infiltration in the affected tissue. ${ }^{1215}$ Interestingly, a recent study by Wei et al demonstrated that $C t l a 4^{+/-} P d c d 1^{-/-}$ mice develop severe myocarditis, which recapitulated ICI-associated myocarditis in humans. ${ }^{16}$ Pathological analysis of the myocardium of these mice demonstrated that the large immune cell infiltrates predominantly consisted of cytotoxic $\mathrm{T}$ cells and macrophages, suggesting that both lymphoid and myeloid immune cells drive inflammation in ICI-associated myocarditis. ${ }^{16}$ Although the majority of acute IRAEs can be overcome by corticosteroid treatment and temporary or permanent discontinuation of ICI therapy, these toxicities may severely compromise the clinical outcome and quality of life of patients with cancer and long-term survivors. ${ }^{317} 18$ In addition to these acute IRAEs, ICI therapy 
may also affect the clinical course of pre-existing autoimmune diseases and other more gradually developing inflammatory conditions. ${ }^{19-21}$ For example, 27\%-75\% of the patients with cancer and a history of rheumatoid arthritis, systemic lupus erythematosus, or inflammatory bowel disease experienced a flare-up on ICI treatment. ${ }^{22-24}$ An important additional concern is the effect of ICIs on atherosclerosis, a chronic lipid-driven inflammatory disease of the larger arteries and a major underlying cause of cardiovascular disease (CVD), including myocardial infarction and ischemic stroke. ${ }^{21}{ }^{25}$ Subclinical atherosclerosis is common and found in $45 \%-75 \%$ of the patients with cancer. ${ }^{26}{ }^{27}$ As cancer and atherosclerosis share several risk factors, such as aging, a sedentary lifestyle, smoking and chronic low-grade inflammation, patients with cancer may be more susceptible to develop clinical complications of atherosclerosis, such as myocardial or cerebral infarction. ${ }^{27}$ Although preclinical studies identified a protective role for the immune checkpoint proteins CTLA4, PD1 and PDL1 in experimental atherosclerosis, clinical data on the effects of ICI-mediated inhibition of these proteins on atherosclerotic CVD were sparse. However, recently, several clinical studies have provided a new perspective on the effects of ICI therapy on atherosclerosis. ${ }^{27-31}$

Here we discuss the effects of ICI therapy on atherosclerosis in patients with cancer and explore the pathophysiology of ICI-related atherosclerotic CVD. Furthermore, we discuss potential strategies to reduce the impact of atherosclerotic CVD on ICI-treated patients with cancer.

\section{ICI THERAPY IS ASSOCIATED WITH ATHEROSCLEROTIC CV EVENTS}

Until recently, clinical studies on the effect of ICIs on atherosclerosis were mainly limited to case reports and smaller cohort studies. ${ }^{27-29} 32$ In a larger study, Bar et al retrospectively analyzed the incidence of acute vascular complications among 1215 patients with cancer who received ICI therapy. ${ }^{30}$ Approximately $1 \%$ of the patients developed a myocardial infarction or ischemic stroke within 6 months after initiation of ICI treatment. ${ }^{30}$ Additionally, a recently published systematic review analyzed the incidence of arterial thrombotic events, in particular stroke and myocardial infarction, following ICI therapy. ${ }^{33}$ Among 17 studies, with a total of 10.106 subjects, the incidence rate of arterial thrombotic events in ICI-treated patients was $1.1 \% .^{33}$ The risk was independent of single or combination ICI treatment, but did seem associated with some cancer types with high thrombogenic properties, such as pancreatic and advanced genitourinary cancer. ${ }^{33}{ }^{34}$ Despite the high level of evidence a systematic review offers, determining the proportion of arterial thrombotic event attributable to ICIs alone remains complex thus far. The included retrospective studies might have suffered from selection bias, and prospective studies had a short follow-up and limited external validity due to their selection criteria. Information on concomitant or previous use of glucocorticoids, chemotherapy and radiotherapy is often not reported. These limitations hamper the translation of these retrospective data to everyday clinical practice.

A recent publication of Drobni et al provides more detailed insights into the association between ICI therapy and atherosclerotic CVD in patients with cancer. ${ }^{31}$ This study investigated the incidence of atherosclerotic $\mathrm{CV}$ events, defined as a composite of myocardial infarction, coronary revascularization, and ischemic stroke, in a cohort of 2842 patients with cancer, with a median age of 64 years who underwent ICI therapy and a control group of patients with cancer who did not undergo ICI treatment. ${ }^{31}$ Non-small cell lung cancer (NSCLC) and melanoma were the most common cancer types in the ICI-treated cohort and the majority of patients $(75.3 \%)$ was treated with PD1 inhibitors, with a median treatment duration of five cycles. Although the control group was matched for age, CV history, and cancer type, the presence of classical CV risk factors, including hypertension, hyperlipidemia and diabetes mellitus, was more common in the control group. ${ }^{31}$ Accordingly, the use of angiotensin receptor blockers, ACE inhibitors, $\beta$-blockers, aspirin, other antiplatelet therapies, and non-statin dyslipidemia therapies was higher in the control group. In this large cohort study, ICI therapy was associated with a 4.7-fold increase in atherosclerotic CV events (5.35 events/100 person years in ICI patients vs 1.02 events / 100 person years in controls). ${ }^{31}$ For the individual secondary outcomes of myocardial infarction, coronary revascularization and ischemic stroke, hazard ratios were 7.2, 3.0, and 4.6, respectively. ${ }^{31} \mathrm{~A}$ limitation of this approach is the potential detection bias that may have occurred due to closer (CV) monitoring of patients following ICI therapy in comparison to the patients who did not receive ICI treatment. The results from the matched cohort study were confirmed with a case-crossover design among the same 2842 patients who started ICI therapy. During a 2-year follow-up period, 119 individuals developed a CV event, as compared with 66 patients in the 2-year period prior to initiation of ICI treatment, resulting in a 4.8-fold increase in $\mathrm{CV}$ events, which confirmed the findings of the case-control study. ${ }^{31}$ Subgroup analysis of the casecontrol study demonstrated that sex, age, Body Mass Index (BMI), a history of CV events, diabetes, and cancer type had no significant impact on the incidence of atherosclerotic CVD. ${ }^{31}$

As atherosclerosis is a pathological process that develops gradually, it may take years or even decades before its clinical complications become manifest. Long-term follow-up studies and registries of ICI-treated patients with cancer, as well as long-term survivors of cancer are therefore required. Nevertheless, the current data demonstrate that ICI therapy is associated with an increased risk of atherosclerotic CVD, even during a limited follow-up period. Importantly, the risk of ICI-related CV complications has so far been reported to be independent of the presence of most classical CV risk factors, such as age, BMI and 
diabetes, which suggests that other pathophysiological factors drive the development of ICI-related atherosclerotic $\mathrm{CV}$ events.

\section{INSIGHTS FROM IMAGING STUDIES: ICI THERAPY INCREASES VASCULAR INFLAMMATION AND PLAQUE PROGRESSION}

Low-grade inflammation of the arterial wall has a critical role in the formation of atherosclerotic lesions and the subsequent development of clinical complications. ${ }^{32}$ Previous studies demonstrated that $2-\left({ }^{18} \mathrm{~F}\right)$ fluorodeoxyglucose (FDG) positron emission tomography (PET)/CT is a valid strategy to evaluate atherosclerosis-associated inflammation in the arterial wall. ${ }^{35}$ As ${ }^{18} \mathrm{~F}$-FDG PET/CT is routinely applied to diagnose malignancies and evaluate therapeutic responses, this imaging modality also provides the opportunity to evaluate the effects of ICI therapy on vascular inflammation in patients with cancer. We previously investigated the effects of short-term ICI therapy on vascular inflammation in a small cohort of 10 ICI-treated patients with melanoma with a mean age of 53 years and without a history of CVD. ${ }^{36}$ The majority of patients was treated with ipilimumab (intratumoral or intravenously) and nivolumab (intravenously), one patient received pembrolizumab (intravenously). FDG uptake was measured in the thoracic aorta and carotid arteries before and 6 weeks after initiation of ICI treatment. No differences in arterial FDG maximum standardized uptake values $\left(\mathrm{SUV}_{\text {max }}\right)$ were observed, indicating that short-term ICI treatment did not affect vascular inflammation in this small cohort of patients with melanoma. ${ }^{36}$ Another recent study investigated the effects of ICIs on vascular inflammation in a group of 20 patients with melanoma, a mean age of 74 years, and unknown history of CVD, who were treated for a longer period. ${ }^{37}$ In this study, in which $80 \%$ of the patients received PD1 inhibitors, $5 \%$ received CTLA4 inhibitors, and $15 \%$ received dual therapy with PD1 and CTLA4 inhibitors, FDG uptake was measured in six arterial regions (ascending aorta, aortic arch, descending aorta, abdominal aorta, and both iliac arteries) before and 4.4 months after initiation of ICI treatment. ${ }^{37}$ ICI therapy significantly increased FDG uptake in all arterial regions, as reflected by a $22.1 \%$ increase in FDG $\mathrm{SUV}_{\max }$ after the start of ICI treatment. ${ }^{37}$ Interestingly, the increase of $\mathrm{SUV}_{\max }$ values was most prominent in noncalcified and mildly calcified arterial segments, whereas FDG uptake in the moderately-severely calcified arterial wall was unaffected by ICI therapy. ${ }^{37}$ These important findings suggest that ICI therapy aggravates inflammation particularly in more initial, non-calcified and mildly calcified atherosclerotic lesions and not in advanced calcified lesions. This corresponds with the histological characteristics of initial atherosclerotic plaques, which have a relatively high abundance of inflammatory cells, whereas advanced lesions contain larger necrotic, calcified and fibrotic regions and relatively lower numbers of immune cells. ${ }^{38}{ }^{39}$ Importantly, these results imply that ICI therapy may accelerate the progression of initial atherosclerotic lesions towards more advanced, clinically unfavorable plaques by increasing vascular inflammation.

In a different approach which used conventional CT imaging to assess the effect of ICIs on thoracic atherosclerotic plaque burden, Drobni et al demonstrated that the progression rate of both total and non-calcified plaque volume increased after ICI therapy. ${ }^{31}$ Thoracic plaque volume increased from $2.1 \%$ /year before ICI treatment to $6.7 \%$ /year following the initiation of ICI therapy. Interestingly, the ICI-associated increase in total plaque burden was significantly lower in patients with cancer who used statins, as compared with patients who did not use these agents, suggesting that statins may reduce the ICIrelated increase in plaque progression, which may have important clinical consequences, as discussed further. ${ }^{31}$ In contrast to these findings, partial resolution of large, but uncalcified, atherosclerotic lesions following anti-PD(L) one treatment has been described in a case report and a small cohort study. ${ }^{40}{ }^{41}$ Unfortunately, the reports did not include PET-CT imaging, which would have improved the interpretation of these interesting observations.

In addition to local vascular inflammation, atherosclerotic disease is also characterized by a systemic inflammatory response, reflected by increased hematopoietic activity and elevated levels of the inflammatory biomarker C reactive protein (CRP) ${ }^{42}{ }^{43}$ Consistent with previous studies, we recently demonstrated that ICIs did not affect FDG uptake in the spleen and vertebral bone marrow of patients with cancer, indicating that hematopoietic activity was unaffected by ICI therapy. ${ }^{36}{ }^{37}$ Moreover, plasma CRP levels were not affected by ICI treatment. ${ }^{37}$ Although these data suggest that short-term ICI therapy does not have a major impact on the atherosclerosis-associated systemic inflammatory response in patients with cancer, more detailed analyses of the number and activation status of circulating immune cells, such as monocytes and T cells, and inflammatory mediators, such as interleukin (IL)-1 $\beta$ and IL-6, will be required to address this important point.

Together these imaging studies demonstrate that ICI therapy not only triggers low-grade inflammation of the arterial wall but also increases the progression rate of atherosclerosis in ICI-treated patients, which may contribute to increased occurrence of acute CV events in these patients.

\section{PATHOPHYSIOLOGY OF ICI-RELATED ATHEROSCLEROTIC CVD}

ICIs release the natural brake on T-cell activation, which enhances T cell-mediated antitumor responses. ${ }^{3}$ Besides these on-target effects, ICI-induced T-cell activation may also elicit off-tumor immune responses that result in acute IRAEs, flare-ups of pre-existing autoimmune diseases, or aggravation of more gradually developing inflammatory conditions, such as atherosclerosis. ${ }^{3}{ }^{19-21}$ Mass cytometry and single-cell RNA sequencing of atherosclerotic lesions recently identified $\mathrm{T}$ cells as a dominant immune cell type in both human and murine atherosclerotic lesions. ${ }^{44-46}$ The phenotypical spectrum of $\mathrm{CD} 4^{+}$and $\mathrm{CD} 8^{+} \mathrm{T}$ cells in 
Table 1 Overview of in vivo experiments determining the role of CTLA4, PD1 and PDL1/2 in atherosclerosis

\begin{tabular}{|c|c|c|c|c|}
\hline Study & Model & Atherosclerosis & Effect on plaque & Effect on immune cells \\
\hline Bu et $a l^{49}$ & $P d 1^{-/-} L_{d l l}^{-/-}$mice & $\uparrow$ & $\begin{array}{l}\uparrow \text { Lesion size } \\
\uparrow \mathrm{CD}^{+} \mathrm{T} \text { cells } \\
\uparrow \mathrm{CD}^{+} \mathrm{T} \text { cells } \\
\uparrow \text { Macrophages } \\
\uparrow \text { Apoptotic cells }\end{array}$ & $\begin{array}{l}\uparrow \text { IFN- } \gamma \text { and TNF- } \alpha \text { in } \\
\text { splenic T cells }\end{array}$ \\
\hline Bu et $a l^{49}$ & $\begin{array}{l}\text { Antibody-mediated PD1 } \\
\text { inhibition in } L d l r^{-/-} \text {mice }\end{array}$ & $\uparrow$ & $\begin{array}{l}=\text { Lesion size } \\
\uparrow \text { CD }^{+} T \text { cells } \\
\uparrow \text { CD8 }^{+} T \text { cells }\end{array}$ & $\begin{array}{l}\uparrow \text { IFN-producing splenic } \mathrm{T} \\
\text { cells } \\
\uparrow \text { Lymph node size } \\
\uparrow \text { Lymph node CD4 }{ }^{+} \text {CD8 } \\
\text { T cells }\end{array}$ \\
\hline
\end{tabular}

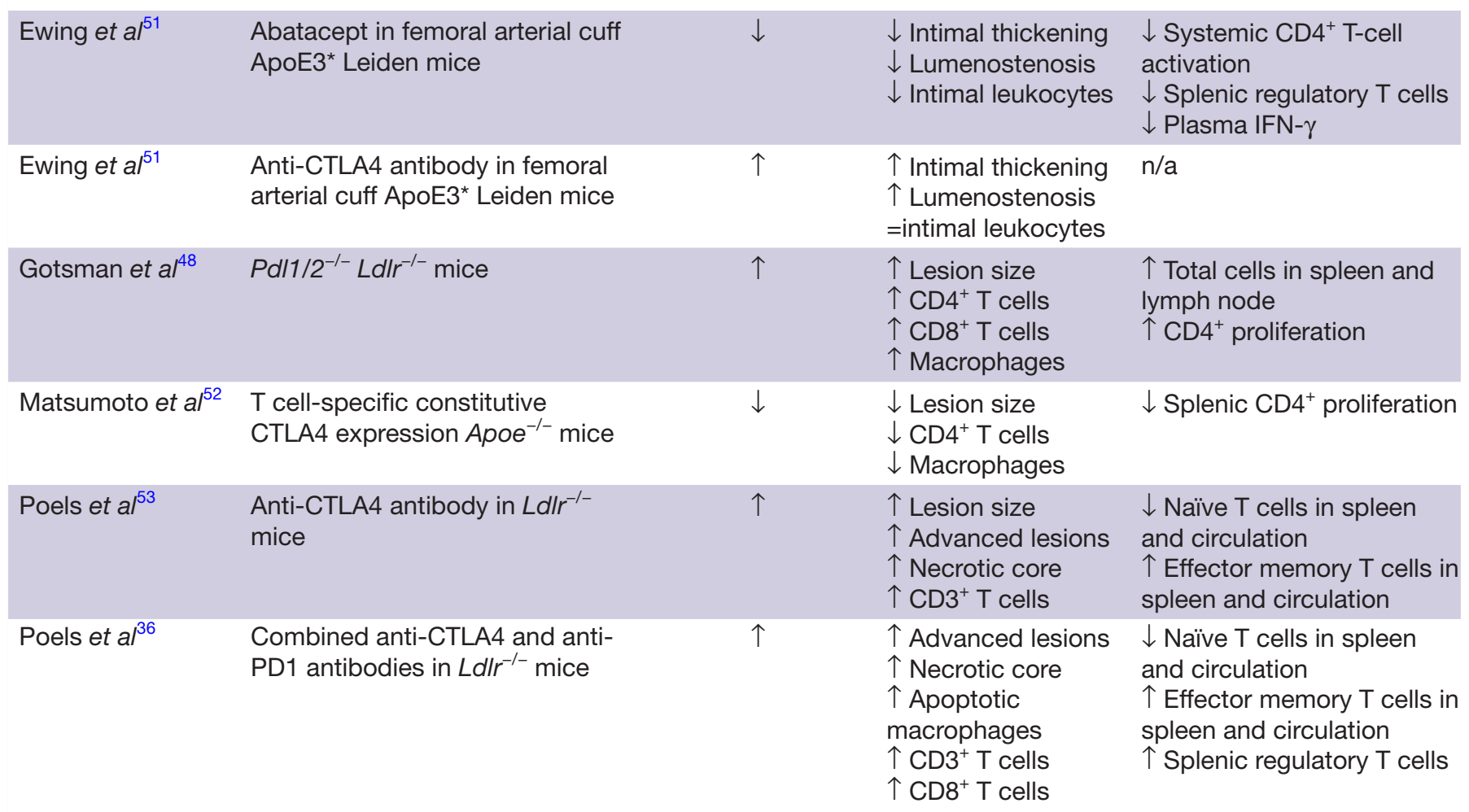

$\uparrow$ indicates an increase in atherosclerotic burden; $\downarrow$ indicates a decrease in atherosclerotic burden.

CTLA4, cytotoxic T lymphocyte-associated protein 4; IFN- $\gamma$, interferon gamma; PD1, programmed cell death protein 1; PDL1, programmed cell death protein ligand 1.

the plaque ranged from a fully activated status, characterized by the expression of inflammatory effector molecules, such as granzymes and cytokines, to a more quiescent and exhausted phenotype. ${ }^{44-46}$ Exhausted T cells are, among other markers, characterized by high PD1 expression, indicating that antibody-mediated blockade of PD1 could reactivate these exhausted cells. ${ }^{46}$ Given their abundant presence and heterogenic phenotype in the atherosclerotic plaque, $\mathrm{T}$ cells not only drive the progression of atherosclerosis towards more advanced, clinically unfavorable lesions but also directly contribute to plaque rupture and the subsequent development of acute $\mathrm{CV}$ events. ${ }^{21} 25444547$ Consequently, ICI-mediated activation of $\mathrm{T}$ cells may have a major impact on atherogenesis.

Genetic knockout models and pharmacological modulation of the ICI-target proteins PD1, PDL1, and CTLA4 have been used to explore the role of these co-inhibitory proteins in experimental atherosclerosis (table 1$){ }^{32}$ Genetic deficiency of PD1, PDL1/2 or antibody-mediated inhibition of PD1 aggravated atherosclerotic burden in hyperlipidemic $\mathrm{Ldlr}^{--}$mice and induced an inflammatory plaque phenotype characterized by an increased abundance of both $\mathrm{CD} 4^{+}$and $\mathrm{CD} 8^{+} \mathrm{T}$ cells and macrophages. ${ }^{48} 49 \mathrm{Pdl1} / 2^{-/-} L d l r^{-/-} \mathrm{T}$ cells were more susceptible to antigen-presenting cell-induced proliferation, had an activated phenotype, characterized by high CD25 and low CD62L expression, and expressed higher levels of the proatherosclerotic cytokines interferon gamma and tumor necrosis factor alpha. ${ }^{49}$ Together these findings demonstrate that deficiency of the PD1-PDL1 dyad triggers an activated T-cell phenotype that drives atherogenesis. A comparable atheroprotective role has been attributed to CTLA4. Both T cell-specific overexpression of CTLA4 or treatment with the CTLA4-Ig fusion protein 


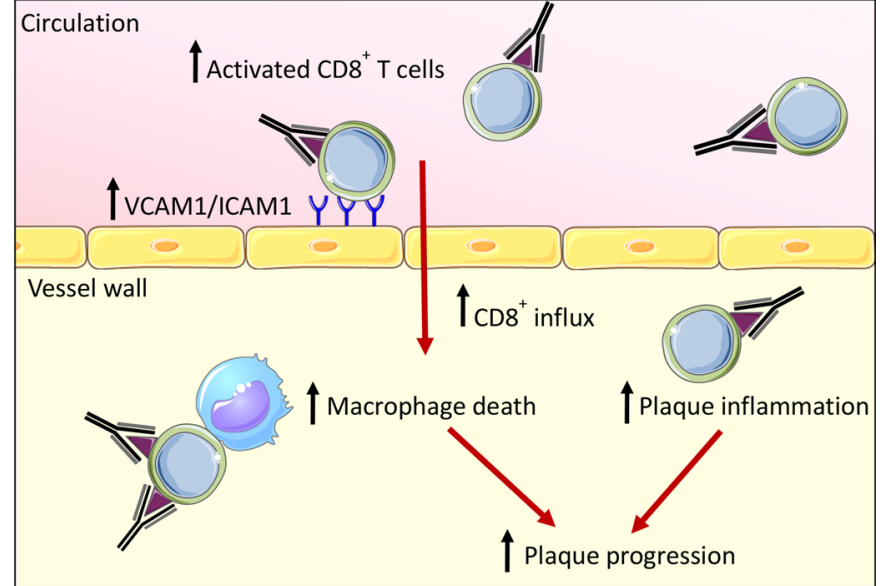

Immune checkpoint inhibitor Macrophage $\bigcirc$ CD8 $^{+}$T cell Y VCAM1/ICAM1

$\triangle$ CTLA4/PD1

Figure 1 Effects of antibody-mediated blockade of CTLA4 and PD1 in Ldlr ${ }^{-/}$mice. Immune checkpoint inhibitors inhibit CTLA4 and PD1 signaling and thereby promote activation of both splenic and circulating $\mathrm{CD}^{+} \mathrm{T}$ cells. Endothelial activation markers VCAM1 and ICAM1 are upregulated and facilitate the influx of $\mathrm{CD}^{+} \mathrm{T}$ cells into the vessel wall. Once they have entered the lesion, $C D 8^{+} \mathrm{T}$ cells induce macrophage death and increase the T-cell:macrophage ratio, thus driving lymphoid-driven plaque inflammation. Together, this promotes plaque progression. CTLA4, cytotoxic $T$ lymphocyte-associated protein 4; PD1, programmed cell death protein 1.

abatacept reduced atherosclerosis in hyperlipidemic mice, whereas antibody-mediated inhibition of CTLA4 increased atherosclerotic burden in $\mathrm{Ldlr}^{-/-}$mice. ${ }^{50-53}$ Blockage of CTLA4 induced an activated CD4 $4^{+}$and $\mathrm{CD}^{+}$ T-cell profile in the circulation and lymphoid organs and promoted activation of the aortic endothelium. ${ }^{53}$ Atherosclerotic lesion size increased on CTLA4 inhibition and plaques contained more $\mathrm{T}$ cells, which promoted necrotic core formation and subsequent plaque progression towards an advanced, clinically unfavorable phenotype. ${ }^{53}$ As combined inhibition of CTLA4 and PD1 is a therapeutic strategy for several malignancies, we recently investigated the effects of dual antibody-mediated inhibition of CTLA4 and PD1 on atherosclerosis in $\mathrm{Ldlr}^{-/-}$mice. ${ }^{36}$

Short-term ( 5 weeks) antibody treatment did not affect atherosclerotic lesion size. However, morphological analysis of the plaque phenotype demonstrated that inhibition of CTLA4 and PD1 resulted in a more advanced, clinically unfavorable plaque phenotype. ${ }^{36}$ Antibody treatment also induced endothelial activation, characterized by increased expression of adhesion molecules, including VCAM1 and ICAM1, which may enhance immune cell migration into the plaque. ${ }^{36}$ Accordingly, $\mathrm{CD}^{+}$T-cell abundance in the plaque increased by 2.7-fold, which increased the $\mathrm{T}$ cell:macrophage ratio (figure 1). ${ }^{36}$ Interestingly, a comparable $\mathrm{T}$ cell-driven inflammatory response was observed in coronary atherosclerotic lesions of 11 ICI-treated patients with cancer who died from non-CV causes. ${ }^{54}$ Together these data highlight an atheroprotective role for PD1, PDL1 and CTLA4 in atherosclerosis and suggest that ICI-mediated inhibition of these proteins induces a predominantly $\mathrm{T}$ cell-driven inflammatory response in atherosclerotic plaques, which promotes lesion formation and progression towards clinically unfavorable plaque phenotypes.

While the molecular pathways of ICI-associated atherosclerosis are incompletely understood, Luoma et al demonstrated that ICI-related colitis, a common and more acute ICI-associated IRAE, was associated with striking changes in $\mathrm{CD}^{+}$and $\mathrm{CD} 4^{+} \mathrm{T}$-cell populations in the colon, characterized by a shift from a tissue-resident memory phenotype to a proliferating, inflammatory effector cell profile. ${ }^{55}$ Additional alterations were also detected in regulatory T-cell populations. ${ }^{55}$ Although regulatory T-cell numbers in the colon were increased in ICI-treated patients with colitis, a cluster of regulatory $\mathrm{T}$ cells that expressed inflammatory genes, such as IL12RB2, CXCR3 and STAT1, was enriched in patients with colitis, suggesting that the regulatory function of these cells was compromised. ${ }^{55}$ Myeloid cells that expressed high levels of cytokines, such as tumour necrosis factor, IL-1 $\beta$ and oncostatin M (OSM), and chemokines (eg. CXCL9 and CXCL10) were also enriched in biopsies from ICI-treated patients with colitis. ${ }^{55}$ Together these data show that ICIinduced colon inflammation results from a complex multidimensional inflammatory network that involves the activation, proliferation and recruitment of effector $\mathrm{T}$ cells, potential suppression of regulatory T-cell function, as well as the activation of myeloid cells. ${ }^{55}$ Whether a comparable inflammatory program also drives more gradually developing ICI-related complications, such as atherosclerotic CVD, should be investigated in future studies.

\section{ROLE OF CONVENTIONAL CV RISK STRATIFICATION IN ICI- ASSOCIATED ATHEROSCLEROTIC CVD}

Besides an etiological approach to elucidate the pathophysiological pathways that drive ICI-associated atherosclerotic CVD, predictive research is warranted for risk stratification and possible prevention of $\mathrm{CV}$ events. Increasing the awareness of ICI-associated atherosclerotic CVD among clinicians is an important first step to prevent these adverse events. However, further risk stratification is required to identify those individuals that may benefit most from preventive measures. Conflicting data have been reported on the role of traditional CV risk factors in the development of ICI-associated atherosclerotic CV events. For example, a retrospective single-center study among 1215 patients with cancer found that the presence of hypertension, dyslipidemia or a history of CVD increased the risk of novel CV events following the initiation of ICI therapy. ${ }^{30}$ Accordingly, the presence of subclinical atherosclerosis (eg, severe coronary calcification or severe aortic calcification) on routinely obtained chest and abdominal CT scans prior to ICI therapy was also associated with ICIrelated CV events. ${ }^{56}$ In contrast, Drobni and colleagues 
found no significant associations between ICI-associated $\mathrm{CV}$ events and sex, age, the presence of type 2 diabetes mellitus or a history of CVD in a cohort of 2842 patients with cancer with matched controls. ${ }^{31}$ Similar findings were reported by Schiffer $e t a l$, who recently analyzed CV events among 76 ICI-treated patients who were referred to a cardio-oncology clinic. ${ }^{56}$ During a median follow-up of 11 months, 80 non-ischemic and ischemic CV events were observed among 49 patients and the median time to an event was 97 days after the start of ICI therapy ${ }^{56}$ In this small cohort, previous CVD and the presence of conventional CV risk factors, including smoking, hypertension, type 2 diabetes mellitus and hyperlipidemia, were not associated with the occurrence of CV complications. ${ }^{56}$ The somewhat unexpected finding of no association between traditional risk factors and CV events in these studies might indicate that there is a risk of bias. To draw the correct inferences, future studies should focus on preventing possible mechanisms of bias that might occur in retrospective studies. Confounding by indication might have occurred because the underlying probability to be prescribed with an ICI (metastatic disease) can also be associated with CVD or because cardioprotective medication associated with being hypertensive (blood pressure medication and/or statins) interacts with ICI, resulting in a lower risk of CV disease (and thereby leading to a less strong association between traditional risk factors and the outcome). Matching is part of the solution but might lead to selection bias. In future studies, competing risk analysis and propensity score matching could be solutions to address confounding and selection in retrospective studies, since the risk of being exposed or not is taken into account, based on patient and treatment variables. Future studies should preferably be prospective cohort studies with detailed information on prior treatment (chemotherapy and radiotherapy), concomitant therapy (glucocorticoids and cardioprotective medication), and with a long follow-up time and clear selection criteria, to address internal and external validity issues.

Risk stratification beyond traditional risk factors can be assessed with non-invasive vascular measurements. For example, the carotid intima-media thickness test relies on the ultrasonic measurement of the thickness between the two inner layers of the blood vessel, caused by intimal hyperplasia in atherosclerosis. ${ }^{57}$ Additionally, the pulse wave velocity (PWV) assesses arterial stiffness by measuring the rate at which a blood pressure wave propagates through the artery. ${ }^{58}$ Both tests are independent risk markers for atherosclerotic disease and especially the PWV has emerged as a useful tool for diagnosis and risk stratification as it offers incremental information in the prediction for future CVD ${ }^{57-59}$ Additionally, a recent study used CT scans to determine calcification levels in the coronary arteries (right, left main, left anterior and left circumflex) of ICI-treated patients and found a positive correlation between baseline coronary calcification and CV events after ICI use $(p=0.018) .{ }^{56}$ Even though no atherosclerotic CVD cases were reported in this small cohort $(\mathrm{n}=76),{ }^{56}$ a similar imaging strategy could be used to identify whether risk of aggravated atherosclerosis can be predicted with baseline CT scans.

Together these studies suggest that additional CV risk stratification strategies may be required to identify the individuals who are at risk of developing CVD following ICI therapy. Whether circulating inflammatory biomarkers, such as CRP, the presence of low-grade vascular inflammation detected on routinely obtained imaging studies, including ${ }^{18}$ F-FDG PET/CT, or the assessment of subclinical atherosclerosis improves $\mathrm{CV}$ risk stratification should be determined in large and long-term follow-up studies of ICI-treated patients with cancer and long-term survivors. These markers need to be further validated in prospective cohorts and risk prediction studies can assess the incremental value that these novel markers offer in risk stratification beyond traditional risk factors.

\section{POTENTIAL PREVENTIVE PHARMACOLOGICAL STRATEGIES}

Hydroxymethylglutaryl-CoA (HMG-CoA) reductase inhibitors, also known as statins, are a well-characterized and broadly applied therapy for dyslipidemia. Interestingly, the concomitant use of statins reduced the annual increase in total atherosclerotic burden among ICI-treated patients with cancer by $\sim 37 \% .{ }^{31}$ The statinassociated reduction was even more pronounced in noncalcified atherosclerotic lesions, indicating that statins may halt the progression of initial atherosclerotic lesions towards clinically unfavorable calcified lesions in these subjects. ${ }^{31}$ These observations are in accordance with previous observations in patients without cancer, in which statins reduced both systemic and local inflammation in the atherosclerotic plaque, thereby improving the stability of these lesions. ${ }^{60}{ }^{61}$ Whether statins have similar beneficial effects in patients without dyslipidemia is currently unknown. Although it is suggested that HMG-CoA reductase inhibitors have the potential to halt the progression of atherosclerosis in ICI-treated patients, large prospective clinical studies are undoubtedly required to further evaluate the efficacy and safety of statins in this context. ${ }^{31}$ Similar to statins, the concomitant short-term use of corticosteroids was also associated with a reduced incidence of ICI-associated CVD among patients with cancer. ${ }^{31}$ However, the adverse effects of these anti-inflammatory drugs, as well as the potential negative effects on ICI efficacy, limit the clinical applicability of corticosteroids as preventive pharmacological intervention in ICI-treated patients with cancer who are at risk of atherosclerotic CVD.

Given the central role of low-grade, subclinical inflammation in atherogenesis, several recent clinical studies evaluated the efficacy of anti-inflammatory interventions on (recurrent) atherosclerotic CVD. For example, lowdose colchicine, an anti-inflammatory drug currently registered for the treatment of gout, familial Mediterranean fever, and pericarditis, reduced ischemic $\mathrm{CV}$ events in patients with coronary artery disease. ${ }^{62}$ 
Moreover, colchicine reduced recurrent ischemic CV events in patients with a recent myocardial infarction. ${ }^{63}$ Whether colchicine has the potential to reduce ICIassociated atherosclerosis is currently unknown, but it has been reported that colchicine prevented recurrent ICI-associated inflammatory arthritis in a patient who received the PD1 inhibitor nivolumab for renal cell carcinoma. ${ }^{64}$ The well-known and favorable safety profile, oral availability and inexpensiveness may further increase the clinical applicability of this drug.

\section{DRUGS TO BOOST ICI EFFICACY?}

Several recent studies suggest a possible interaction between conventional CV drugs and the efficacy of ICIs. In a retrospective observational study, Cortellini et al assessed the impact of the concomitant use of several frequently prescribed drugs for CVD on clinical outcome of 1012 anti-PD(L)1-treated patients with cancer, in particular NSCLC, melanoma and renal cell carcinoma. ${ }^{65}$ After a median follow-up of 24.2 months, it was found that the use of either $\beta$-blockers (HR 1.76, 95\% CI 1.16 to 2.69), statins (HR 1.60, 95\% CI 1.14 to 2.25) and aspirin (HR 1.47, 95\% CI 1.04 to 2.08) was associated with an increased response rate to ICI therapy. ${ }^{65}$ Although additional studies are required to confirm these observational data, previous (pre)clinical studies also showed that conventional CV drugs may affect ICI efficacy. ${ }^{66-68}$ For example, the nonselective $\beta$-blocker propranolol increased progressionfree survival in patients with stage IB-IIIa melanoma in a small prospective study. ${ }^{66}$ In a murine melanoma model, both non-selective $\beta$-blockers and selective beta-2 adrenergic antagonists improved antitumor immunity following PD1 inhibition, potentially by enhancing both T-cell and myeloid effector functions. ${ }^{67}$ Statin treatment also improved the efficacy of PD1 targeted therapies in murine cancer models. ${ }^{69}$ Moreover, cholesterol has been shown to compromise antitumor activity of cytotoxic T cells by increasing ER stress and inducing the expression of co-inhibitory molecules, such as PD1, LAG3 and TIM3. ${ }^{70}$ Accordingly, reduction of cholesterol restored the antitumor activity of these cells. ${ }^{70}$ Recently, it was also demonstrated that antibody-mediated inhibition of the enzyme proprotein convertase subtilisine/kexine type 9 , which is a recently implemented therapy for dyslipidemia, augments the efficacy of ICI therapy in murine melanoma models by increasing the expression of MHC class I on tumor cells, which increased the immunogenicity of the tumor. ${ }^{68}$ These clinical and preclinical observations, in conjunction with many other studies, suggest that conventional $\mathrm{CV}$ drugs may enhance the efficacy of ICI treatment. This important concept warrants further investigation, especially as these CV drugs are readily available, have known safety profiles and could therefore be rapidly implemented as additive strategy to boost ICI efficacy.

\section{CONCLUSIONS AND FUTURE PERSPECTIVE}

In this review, we discussed recently published studies demonstrating increased incidence of atherosclerotic CVD in ICI-treated patients. As ICIs are being increasingly prescribed, the number of patients with ICI-associated atherosclerotic CVD is expected to rise in the coming years. Therefore, it is of vital importance to unravel the mechanisms that drive ICI-induced alterations in atherosclerosis. Additionally, the use of conventional CV drugs to counteract ICI's detrimental effects should be investigated. So far, conventional CV risk factors have not been able to identify at-risk patients of ICI-associated atherosclerosis. Identification of novel (proinflammatory) biomarkers which can distinguish at-risk populations could greatly improve the prospects of cancer survivors in the future but are probably hard to find due to confounding generation of tumor-related proinflammatory mediators. We would therefore highly recommend to go beyond classical risk assessment and implement non-traditional risk stratification parameters, especially (advanced) imaging strategies, such as intima-media thickness (IMT) or PWV, but also coronary CT, to assess aggravated atherosclerosis and identify at-risk patients.

To improve our knowledge on the scale and severity of ICI-associated atherosclerotic CVD, it is essential to increase awareness among all physicians that encounter either current receivers of ICI therapy or long-term cancer survivors. By actively monitoring these (ex-) patients, steps can be made towards a registry where data on the history, stadium, propensity score, course of treatment and IRAEs, and their pathophysiology can be collected. Moreover, meta-analyses of specific subgroups of patients, for example, those who previously underwent chemotherapy or radiation therapy, those with pre-existing CVD risk factors or those on CV medication, could aid in the riskstratification of these patients. Additionally, long-term effects could become evident when patients are followed up for an extended time period and the efficiency of treatment options could be analyzed to optimize qualityof-life of these survivors. Until then, optimal risk assessment strategies and optimal clinical surveillance for CVD are paramount to reduce atherosclerotic CVD-associated mortality and morbidity in ICI-treated patients.

\section{Author affiliations}

${ }^{1}$ Department of Medical Biochemistry, Amsterdam Cardiovascular Sciences (ACS), Amsterdam UMC, Location AMC, University of Amsterdam, Amsterdam, Netherlands

${ }^{2}$ Department of Psychosocial Research and Epidemiology (PSOE), Netherlands Cancer Institute, Amsterdam, Netherlands

${ }^{3}$ Department of Hematology, Amsterdam UMC, University of Amsterdam, Amsterdam, Cancer Center Amsterdam and LYMMCARE, Amsterdam, Netherlands ${ }^{4}$ Department of Cardiology, Heart Lung Centre, Leiden University Medical Centre, Leiden, Netherlands

${ }^{5}$ Institute for Cardiovascular Prevention (IPEK), Ludwig Maximilian's University, Munich, Germany

${ }^{6}$ German Centre for Cardiovascular Research (DZHK), Partner Site Munich Heart Alliance, Munich, Germany

${ }^{7}$ Department of Medical Oncology, Antoni van Leeuwenhoek - Netherlands Cancer Institute, Amsterdam, Netherlands 
Contributors All authors contributed to the design and drafting of the manuscript.

Funding This study was funded by The Rebrandt Institute of Cardiovascular Science (Rembrandt Grant 2020); Cancer Center Amsterdam (CCA grant 2020).

Competing interests None declared.

Patient consent for publication Not required.

Provenance and peer review Not commissioned; externally peer reviewed.

Open access This is an open access article distributed in accordance with the Creative Commons Attribution Non Commercial (CC BY-NC 4.0) license, which permits others to distribute, remix, adapt, build upon this work non-commercially, and license their derivative works on different terms, provided the original work is properly cited, appropriate credit is given, any changes made indicated, and the use is non-commercial. See http://creativecommons.org/licenses/by-nc/4.0/.

\section{REFERENCES}

1 Hodi FS, Chiarion-Sileni V, Gonzalez R, et al. Nivolumab plus ipilimumab or nivolumab alone versus ipilimumab alone in advanced melanoma (CheckMate 067): 4-year outcomes of a multicentre, randomised, phase 3 trial. Lancet Oncol 2018;19:1480-92.

2 Larkin J, Chiarion-Sileni V, Gonzalez R, et al. Five-Year survival with combined nivolumab and ipilimumab in advanced melanoma. $N$ Engl J Med 2019;381:1535-46.

3 Postow MA, Sidlow R, Hellmann MD. Immune-Related adverse events associated with immune checkpoint blockade. $N$ Engl J Med 2018;378:158-68.

4 Haslam A, Prasad V. Estimation of the percentage of US patients with cancer who are eligible for and respond to checkpoint inhibitor immunotherapy drugs. JAMA Netw Open 2019;2:e192535.

5 Herbst RS, Giaccone G, de Marinis F, et al. Atezolizumab for first-line treatment of PD-L1-Selected patients with NSCLC. N Engl J Med 2020;383:1328-39.

6 Mittendorf EA, Zhang $\mathrm{H}$, Barrios $\mathrm{CH}$, et al. Neoadjuvant atezolizumab in combination with sequential nab-paclitaxel and anthracyclinebased chemotherapy versus placebo and chemotherapy in patients with early-stage triple-negative breast cancer (IMpassion031): a randomised, double-blind, phase 3 trial. Lancet 2020;396:1090-100.

7 Galsky MD, Arija José Ángel Arranz, Bamias A, et al. Atezolizumab with or without chemotherapy in metastatic urothelial cancer (IMvigor130): a multicentre, randomised, placebo-controlled phase 3 trial. Lancet 2020;395:1547-57.

8 Gutzmer R, Stroyakovskiy D, Gogas H, et al. Atezolizumab, vemurafenib, and cobimetinib as first-line treatment for unresectable advanced BRAF ${ }^{\mathrm{V} 600}$ mutation-positive melanoma (IMspire150): primary analysis of the randomised, double-blind, placebocontrolled, phase 3 trial. Lancet 2020;395:1835-44.

9 Powles T, Park SH, Voog E, et al. Avelumab maintenance therapy for advanced or metastatic urothelial carcinoma. $N$ Engl J Med 2020;383:1218-30.

10 Baxi S, Yang A, Gennarelli RL, et al. Immune-related adverse events for anti-PD-1 and anti-PD-L1 drugs: systematic review and metaanalysis. BMJ 2018;360:k793.

11 Shankar B, Zhang J, Naqash AR, et al. Multisystem immune-related adverse events associated with immune checkpoint inhibitors for treatment of non-small cell lung cancer. JAMA Oncol 2020;6:1952-6.

12 Salem J-E, Manouchehri A, Moey M, et al. Cardiovascular toxicities associated with immune checkpoint inhibitors: an observational, retrospective, pharmacovigilance study. Lancet Oncol 2018;19:1579-89.

13 Escudier M, Cautela J, Malissen N, et al. Clinical features, management, and outcomes of immune checkpoint Inhibitor-Related cardiotoxicity. Circulation 2017;136:2085-7.

14 Wang DY, Salem J-E, Cohen JV, et al. Fatal toxic effects associated with immune checkpoint inhibitors: a systematic review and metaanalysis. JAMA Oncol 2018;4:1721-8.

15 Lindquist M. VigiBase, the who global ICSR database system: basic facts. Drug Inf J 2008;42:409-19.

16 Wei SC, Meijers WC, Axelrod ML, et al. A genetic mouse model recapitulates immune checkpoint inhibitor-associated myocarditis and supports a mechanism-based therapeutic intervention. Cancer Discov 2021;11:614-25.

17 Patrinely JR, Young AC, Quach $\mathrm{H}$, et al. Survivorship in immune therapy: Assessing toxicities, body composition and health-related quality of life among long-term survivors treated with antibodies to programmed death-1 receptor and its ligand. Eur J Cancer 2020;135:211-20.
18 O'Reilly A, Hughes P, Mann J, et al. An immunotherapy survivor population: health-related quality of life and toxicity in patients with metastatic melanoma treated with immune checkpoint inhibitors. Support Care Cancer 2020;28:561-70.

19 Coureau M, Meert A-P, Berghmans T, et al. Efficacy and toxicity of immune -Checkpoint inhibitors in patients with preexisting autoimmune disorders. Front Med 2020;7:137.

20 Gremese E, Alivernini S, Ferraccioli ES, et al. Checkpoint inhibitors (CPI) and autoimmune chronic inflammatory diseases (ACIDs): tolerance and loss of tolerance in the occurrence of immunorheumatologic manifestations. Clin Immunol 2020;214:108395.

21 Lutgens E, Seijkens TTP. Cancer patients receiving immune checkpoint inhibitor therapy are at an increased risk for atherosclerotic cardiovascular disease. J Immunother Cancer 2020;8:e000300.

22 Johnson DB, Sullivan RJ, Ott PA, et al. Ipilimumab therapy in patients with advanced melanoma and preexisting autoimmune disorders. JAMA Oncol 2016;2:234-40.

23 Menzies AM, Johnson DB, Ramanujam S, et al. Anti-Pd-1 therapy in patients with advanced melanoma and preexisting autoimmune disorders or major toxicity with ipilimumab. Ann Oncol 2017;28:368-76.

24 Tison A, Quéré G, Misery L, et al. Safety and efficacy of immune checkpoint inhibitors in patients with cancer and preexisting autoimmune disease: a nationwide, multicenter cohort study. Arthritis Rheumatol 2019;71:2100-11.

25 Seijkens TTP, Lutgens E. Cardiovascular oncology: exploring the effects of targeted cancer therapies on atherosclerosis. Curr Opin Lipidol 2018;29:381-8.

26 Cuddy S, Payne DL, Murphy DJ, et al. Incidental coronary artery calcification in cancer imaging. JACC CardioOncol 2019;1:135-7.

27 Vincent L, Leedy D, Masri SC, et al. Cardiovascular disease and cancer: is there increasing overlap? Curr Oncol Rep 2019;21:47.

28 Tomita Y, Sueta D, Kakiuchi Y, et al. Acute coronary syndrome as a possible immune-related adverse event in a lung cancer patient achieving a complete response to anti-PD-1 immune checkpoint antibody. Ann Oncol 2017;28:2893-5.

$29 \mathrm{Hu}$ Y-B, Zhang Q, Li H-J, et al. Evaluation of rare but severe immune related adverse effects in PD-1 and PD-L1 inhibitors in non-small cel lung cancer: a meta-analysis. Transl Lung Cancer Res 2017;6:S8-20.

30 Bar J, Markel G, Gottfried T, et al. Acute vascular events as a possibly related adverse event of immunotherapy: a single-institute retrospective study. Eur J Cancer 2019;120:122-31.

31 Drobni ZD, Alvi RM, Taron J, et al. Association between immune checkpoint inhibitors with cardiovascular events and atherosclerotic plaque. Circulation 2020;142:2299-311.

32 Kusters PJH, Lutgens E, Seijkens TTP. Exploring immune checkpoints as potential therapeutic targets in atherosclerosis. Cardiovasc Res 2018;114:368-77.

33 Solinas C, Saba L, Sganzerla P, et al. Venous and arterial thromboembolic events with immune checkpoint inhibitors: a systematic review. Thromb Res 2020;196:444-53.

34 Abdol Razak NB, Jones G, Bhandari M, et al. Cancer-Associated thrombosis: an overview of mechanisms, risk factors, and treatment. Cancers 2018;10. doi:10.3390/cancers10100380. [Epub ahead of print: 1110 2018].

35 Tarkin JM, Joshi FR, Rudd JHF. Pet imaging of inflammation in atherosclerosis. Nat Rev Cardiol 2014;11:443-57.

36 Poels K, van Leent MMT, Boutros C, et al. Immune checkpoint inhibitor therapy aggravates $\mathrm{T}$ Cell-Driven plaque inflammation in atherosclerosis. JACC CardioOncol 2020;2:599-610.

37 Calabretta R, Hoeller C, Pichler V, et al. Immune checkpoint inhibitor therapy induces inflammatory activity in large arteries. Circulation 2020;142:2396-8.

38 Jonasson L, Holm J, Skalli O, et al. Regional accumulations of T cells, macrophages, and smooth muscle cells in the human atherosclerotic plaque. Arteriosclerosis 1986;6:131-8.

39 Rohm I, Atiskova Y, Drobnik S, et al. Decreased regulatory T cells in vulnerable atherosclerotic lesions: imbalance between pro- and anti-inflammatory cells in atherosclerosis. Mediators Inflamm 2015;2015:1-13.

40 Lamberti G, Gelsomino F, Brocchi S, et al. New disappearance of complicated atheromatous plaques on rechallenge with PD-1/PD-L1 axis blockade in non-small cell lung cancer patient: follow up of an unexpected event. Ther Adv Med Oncol 2020;12:1758835920913801.

41 Gelsomino F, Fiorentino M, Zompatori M, et al. Programmed death-1 inhibition and atherosclerosis: can nivolumab vanish complicated atheromatous plaques? Ann Oncol 2018;29:284-6.

42 Puri R, Nissen SE, Libby P, et al. C-Reactive protein, but not low-density lipoprotein cholesterol levels, associate with coronary 
atheroma regression and cardiovascular events after maximally intensive statin therapy. Circulation 2013;128:2395-403.

43 van der Valk FM, Kuijk C, Verweij SL, et al. Increased haematopoietic activity in patients with atherosclerosis. Eur Heart $J$ 2017:38:425-32.

44 Fernandez DM, Rahman AH, Fernandez NF, et al. Single-Cell immune landscape of human atherosclerotic plaques. Nat Med 2019;25:1576-88.

45 Depuydt MAC, Prange KHM, Slenders L, et al. Microanatomy of the human atherosclerotic plaque by single-cell transcriptomics. Circ Res 2020;127:1437-55.

46 Winkels $\mathrm{H}$, Ehinger $\mathrm{E}$, Vassallo $\mathrm{M}$, et al. Atlas of the immune cell repertoire in mouse atherosclerosis defined by single-cell RNAsequencing and mass cytometry. Circ Res 2018;122:1675-88.

47 Padgett LE, Araujo DJ, Hedrick CC, et al. Functional crosstalk between $T$ cells and monocytes in cancer and atherosclerosis. $J$ Leukoc Biol 2020;108:297-308.

48 Gotsman I, Grabie N, Dacosta R, et al. Proatherogenic immune responses are regulated by the PD-1/PD-L pathway in mice. J Clin Invest 2007;117:2974-82.

49 Bu D-xiu, Tarrio M, Maganto-Garcia E, et al. Impairment of the programmed cell death-1 pathway increases atherosclerotic lesion development and inflammation. Arterioscler Thromb Vasc Biol 2011;31:1100-7.

50 Buono C, Pang H, Uchida Y, et al. B7-1/B7-2 costimulation regulates plaque antigen-specific T-cell responses and atherogenesis in low-density lipoprotein receptor-deficient mice. Circulation 2004;109:2009-15

51 Ewing MM, Karper JC, Abdul S, et al. T-Cell co-stimulation by CD28-CD80/86 and its negative regulator CTLA-4 strongly influence accelerated atherosclerosis development. Int J Cardiol 2013;168:1965-74

52 Matsumoto T, Sasaki N, Yamashita T, et al. Overexpression of cytotoxic T-Lymphocyte-Associated antigen-4 prevents atherosclerosis in mice. Arterioscler Thromb Vasc Biol 2016;36:1141-51.

53 Poels K, van Leent MMT, Reiche ME, et al. Antibody-Mediated inhibition of CTLA4 aggravates atherosclerotic plaque inflammation and progression in hyperlipidemic mice. Cells 2020;9. doi:10.3390/ cells9091987. [Epub ahead of print: 2908 2020].

54 Newman JL, Stone JR. Immune checkpoint inhibition alters the inflammatory cell composition of human coronary artery atherosclerosis. Cardiovasc Pathol 2019;43:107148.

55 Luoma AM, Suo S, Williams HL, et al. Molecular pathways of colon inflammation induced by cancer immunotherapy. Cell 2020;182:655-71.

56 Schiffer WB, Deych E, Lenihan DJ, et al. Coronary and aortic calcification are associated with cardiovascular events on immune checkpoint inhibitor therapy. Int J Cardiol 2021;322:177-82.
57 Lorenz MW, Markus HS, Bots ML, et al. Prediction of clinical cardiovascular events with carotid intima-media thickness: a systematic review and meta-analysis. Circulation 2007;115:459-67.

58 Vlachopoulos C, Aznaouridis K, Stefanadis C. Prediction of cardiovascular events and all-cause mortality with arterial stiffness: a systematic review and meta-analysis. J Am Coll Cardiol 2010;55:1318-27.

59 Ben-Shlomo Y, Spears M, Boustred C, et al. Aortic pulse wave velocity improves cardiovascular event prediction: an individual participant meta-analysis of prospective observational data from 17,635 subjects. J Am Coll Cardiol 2014;63:636-46.

60 Kwon O, Kang S-J, Kang SH, et al. Relationship between serum inflammatory marker levels and the dynamic changes in coronary plaque characteristics after statin therapy. Circ Cardiovasc Imaging $2017 ; 10$.

61 Antonopoulos AS, Margaritis M, Lee R, et al. Statins as antiinflammatory agents in atherogenesis: molecular mechanisms and lessons from the recent clinical trials. Curr Pharm Des 2012;18:1519-30.

62 Nidorf SM, Fiolet ATL, Mosterd A, et al. Colchicine in patients with chronic coronary disease. N Engl J Med 2020;383:1838-47.

63 Tardif J-C, Kouz S, Waters DD, et al. Efficacy and safety of low-dose colchicine after myocardial infarction. N Engl J Med 2019;381:2497-505.

64 Kim ST, Bittar M, Kim HJ, et al. Recurrent pseudogout after therapy with immune checkpoint inhibitors: a case report with immunoprofiling of synovial fluid at each flare. J Immunother Cancer 2019;7:126.

65 Cortellini A, Tucci M, Adamo V, et al. Integrated analysis of concomitant medications and oncological outcomes from PD-1/PDL1 checkpoint inhibitors in clinical practice. J Immunother Cancer 2020;8:e001361.

66 De Giorgi V, Grazzini M, Benemei S, et al. Propranolol for off-label treatment of patients with melanoma: results from a cohort study. JAMA Oncol 2018;4:e172908.

67 Kokolus KM, Zhang Y, Sivik JM, et al. Beta blocker use correlates with better overall survival in metastatic melanoma patients and improves the efficacy of immunotherapies in mice. Oncoimmunology 2018;7:e1405205.

68 Liu X, Bao X, Hu M, et al. Inhibition of PCSK9 potentiates immune checkpoint therapy for cancer. Nature 2020;588:693-8.

69 Xia Y, Xie Y, Yu Z, et al. The mevalonate pathway is a druggable target for vaccine adjuvant discovery. Cell 2018;175:1059-73.

70 Yang W, Bai Y, Xiong Y, et al. Potentiating the antitumour response of $C D 8\left(^{+}\right) T$ cells by modulating cholesterol metabolism. Nature 2016;531:651-5.

$71 \mathrm{Ma} \mathrm{X}, \mathrm{Bi}$ E, Lu Y, et al. Cholesterol Induces CD8 ${ }^{+} \mathrm{T}$ Cell Exhaustion in the Tumor Microenvironment. Cell Metab 2019;30:143-56. 